\title{
Radiation-induced hepatitis masquerading as metastatic disease: the importance of correlating diagnostic imaging with treatment planning
}

\author{
Arthur A. Parsee ${ }^{1}$, Jordan A. McDonald ${ }^{2}$, Kun Jiang ${ }^{3}$, Kujtim Latifi ${ }^{4}$, Rutika Mehta ${ }^{5}$ Jessica M. Frakes ${ }^{4}$, \\ Jose M. Pimiento ${ }^{6}$, Sarah E. Hoffe ${ }^{4}$ \\ ${ }^{1}$ Department of Radiology, Moffitt Cancer Center, Tampa, FL, USA; ${ }^{2}$ Morsani College of Medicine, University of South Florida, Tampa, FL, USA; \\ ${ }^{3}$ Department of Pathology, ${ }^{4}$ Department of Radiation Oncology, ${ }^{5}$ Department of Gastrointestinal Oncology, ${ }^{6}$ Department of Surgical Oncology, \\ Moffitt Cancer Center, Tampa, FL, USA \\ Correspondence to: Arthur A. Parsee. Department of Radiology, Moffitt Cancer Center, 12902 USF Magnolia Dr, Tampa, FL 33612, USA. \\ Email: arthur.parsee@moffit.org.
}

\begin{abstract}
We are presenting a 63-year-old Caucasian male who complained of 2 months of progressive dysphagia. Upper endoscopy discovered a mass in the distal esophagus near the gastroesophageal junction. Biopsy was consistent with adenocarcinoma. Endoscopic ultrasound (EUS) showed extension beyond the muscularis propria, with an enlarged paraesophageal lymph node (T3N1). Initial positron emission tomography (PET)/computed tomography (CT) showed hypermetabolic portocaval lymphadenopathy presumed to be metastatic, but otherwise without distant disease extension. Neoadjuvant treatment included induction FOLFOX followed by 5,600 cGy over 28 fractions in combination with 5-FU and oxaliplatin. Approximately 3.5 weeks after completion, a repeat PET/CT revealed reduced uptake in both the primary esophageal mass and regional lymph nodes. Of note there were several new mass-like foci of hypermetabolism in the liver, specifically the left lobe, concerning for metastatic disease. Image-guided biopsy did not show any identifiable lesions, but sampling was performed based on anatomical landmarks. Pathology revealed benign parenchyma with minimal inflammation and mild reactive regeneration. In light of this, the patient proceeded to undergo definitive resection via robotic Ivor-Lewis esophagectomy with only 1 positive lymph node. Given pleural involvement by the tumor, staging was revised to pT4aN1 with final histology characterized as adenosquamous carcinoma. Postoperative course was fairly uneventful, with a mild exacerbation of his chronic heart failure. The patient was discharged on post-operative day 7 , with his feeding tube removed at his 2 -week post-operative clinic visit. This scenario is of particular educational value from the standpoint that when the post-treatment PET/CT images are registered to the radiotherapy treatment planning CT and dose, the areas of abnormal uptake in the liver fall within the higher dose regions. Given this and the liver biopsy findings, caution should be exercised before declaring progressive disease following radiotherapy without first reviewing the treatment plan.
\end{abstract}

Keywords: Esophageal adenocarcinoma; neoadjuvant chemoradiation; radiation-induced hepatitis; diagnostic pitfalls; metastatic mimickers

Submitted Jul 15, 2019. Accepted for publication Sep 10, 2019.

doi: 10.21037/jgo.2019.09.09

View this article at: http://dx.doi.org/10.21037/jgo.2019.09.09

\section{Introduction}

Preoperative chemoradiation improved survival in patients with locally advanced esophageal cancer (EC) compared to surgical resection alone in the randomized CROSS trial (1). Initial EC staging consists of fusion positron emission tomography (PET)/computed tomography (CT) in combination with endoscopic ultrasound (EUS) and CT of the chest, abdomen and pelvis. Following preoperative 

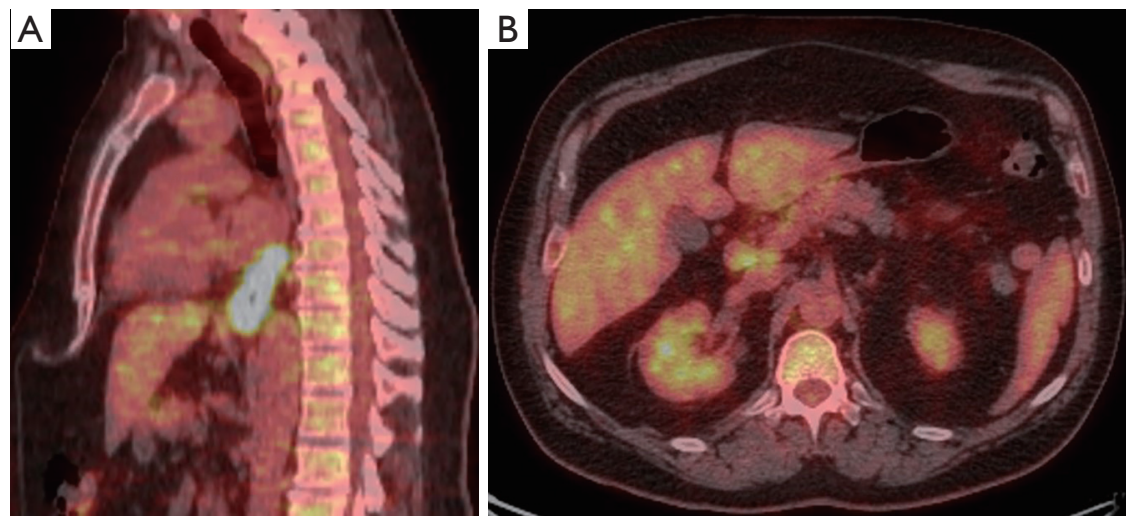

Figure 1 PET/CT sagittal (A) and axial (B) of esophageal carcinoma. (A) Initial PET/CT with long-segment distal esophageal thickening (max SUV 18.3); (B) FDG-avid regional lymphadenopathy (partially-displayed), largest portocaval (max SUV 4.3). Note normal background liver activity. PET/CT, positron emission tomography/computed tomography; SUV, standard uptake value.

therapy, a repeat PET/CT provides quantifiable metrics for assessing residual or new disease, assessing both the size and radiotracer uptake of 18 -fluorodeoxyglucose (FDG) measured against background activity, known as standard uptake value (SUV).

\section{Case presentation}

A 63-year-old Caucasian male presented to his primary care physician with a 2-month history of odynophagia and progressive dysphagia, but without weight-loss or loss of appetite. He consumed alcohol rarely and quit smoking for 10 years following a 20 pack-year history. Occupationally, he had exposure to uranium for 22 years while working at a nuclear power facility. After upper endoscopy, he was found to have a friable mass $38-41 \mathrm{~cm}$ from the incisors, approaching the gastroesophageal junction. Biopsy returned adenocarcinoma. EUS showed tumor extension beyond the muscularis propria as well as an enlarged para-esophageal lymph node, staged T3N1.

Following referral to our tertiary cancer treatment center, an initial PET/CT (Figure 1A,B) illustrated longsegment distal esophageal thickening with hypermetabolism consistent with the primary cancer, as well as portocaval lymphadenopathy that was felt to be metastatic based on size and FDG-uptake. No metastasis was noted in the liver or elsewhere. Only patients who had T1N1 or T2$3 \mathrm{~N} 0-1$ were included on the CROSS trial so the tumor board recommendation for this patient with M1 disease (clinical stage IV) was systemic chemotherapy followed by consideration of local therapies pending response.
After 5 doses of induction FOLFOX chemotherapy, the patient reported some improvement of his dysphagia post treatment, with mild epigastric discomfort. A postchemotherapy PET/CT showed a $>35 \%$ reduction in SUV max, suggesting that the patient would benefit from continuation of the regimen during local irradiation (2). He received 5-fluorouracil and oxaliplatin concurrently with intensity modulated radiation therapy (IMRT) to all initially involved sites. Based on our institutional experience, we focally escalated the dose to the gross tumor to $56 \mathrm{~Gy}$ (3).

Post chemoradiation at 3.5 weeks, a repeat PET/CT (Figure $2 A, B$ ) showed a reduction in the length and FDGuptake of the esophageal primary, as well as normalization of the previously hypermetabolic lymphadenopathy. The imaging timing was chosen to allow for the optimal operative window for maximum benefit, which is generally accepted as 5-8 weeks. Of note, there were several new mass-like foci of hypermetabolism in the medial and lateral left hepatic lobes, but without a correlating CT abnormality.

As the possibility of new metastatic disease would greatly impact the current treatment plan, a multidisciplinary review felt an image-guided liver biopsy would ultimately be needed, given the potential for false negative advanced imaging such as multi-phasic CT or MRI. Neither ultrasound nor contrast-enhanced CT could identify any lesions, so the region of greatest FDG-uptake was targeted based on anatomic landmarks. Biopsy revealed no neoplasm but mild reactive inflammation (Figure 3). Liver function tests had remained stable throughout the pre-operative course. In light of the neoadjuvant treatment response, the patient underwent surgical resection with curative intent. 

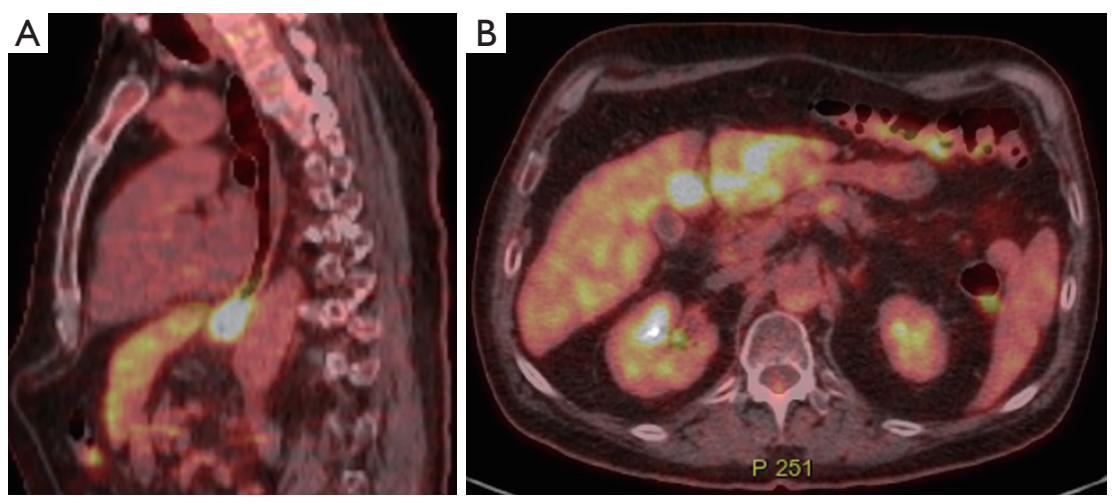

Figure 2 PET/CT sagittal (A) and axial (B) of esophageal carcinoma. (A) Repeat PET/CT with reduced extent of esophageal thickening and decreased hypermetabolism (max SUV 12.2); (B) resolved uptake in regional lymph nodes. New mass-like uptake in the left hepatic lobe (max SUV 7.2). PET/CT, positron emission tomography/computed tomography; SUV, standard uptake value.
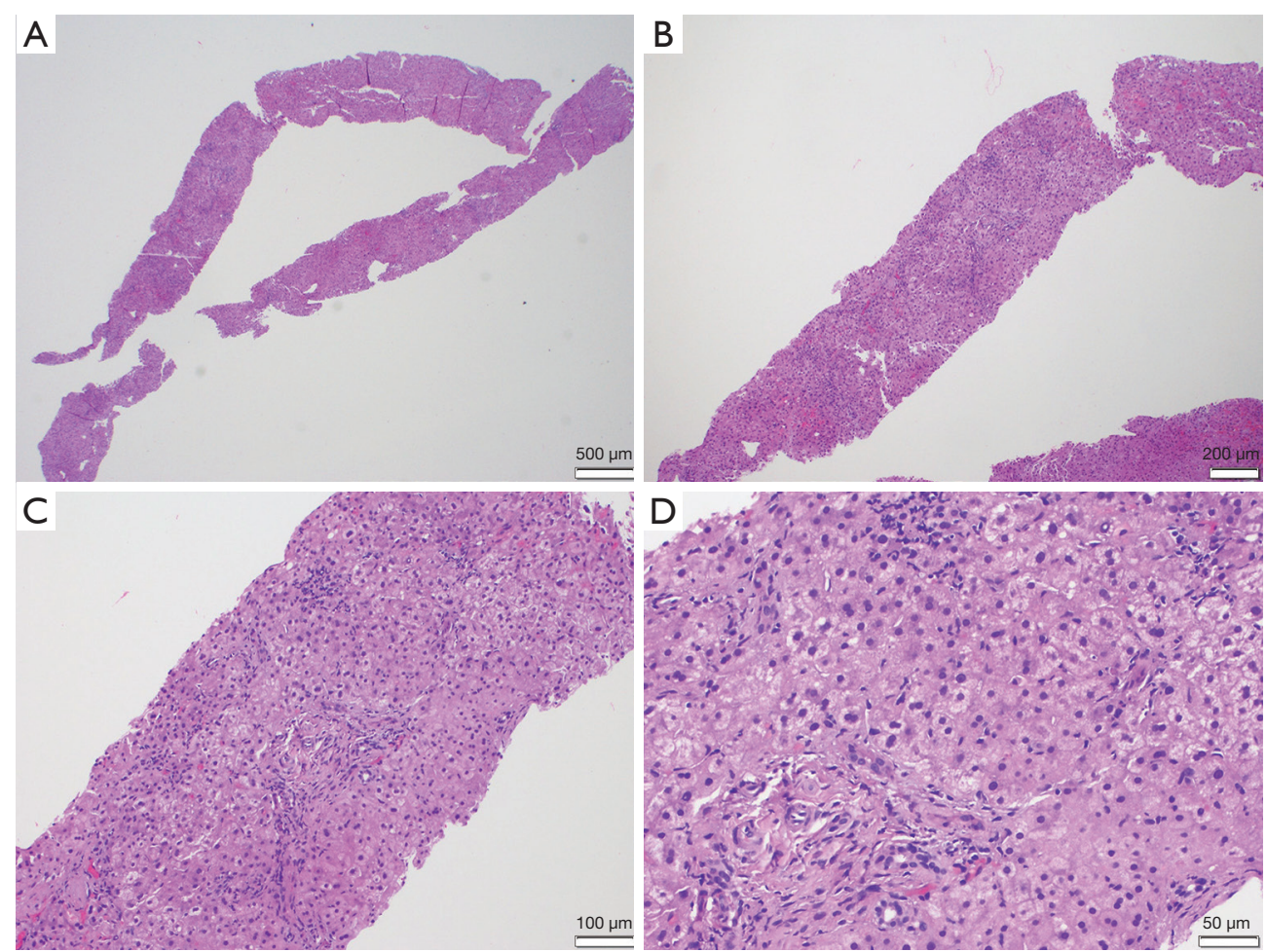

Figure 3 Liver biopsy targeting a "mass lesion". Low- and high-power examination show benign liver parenchyma with mild regeneration, occasional foci of subtle lobular and portal inflammation appreciated; no steatosis, steatohepatitis or fibrosis. Most importantly, no neoplasm or carcinoma. Findings are reactive in nature (A,B,C,D: H\&E sections, $2 \times, 4 \times, 10 \times$ and 20×, respectively).

Robotic-assisted laparoscopic Ivor-Lewis esophagectomy was performed, with initial visual inspection of the liver finding two areas of moderate suspicion. Wedge biopsies yielded negative findings prior to proceeding. Portocaval and hepatic artery nodes were also resected, with placement of a feeding tube. The tumor measured $3.7 \mathrm{~cm} \times 3.4 \mathrm{~cm} \times$ $3.4 \mathrm{~cm}$ with pleural involvement and 1 of 39 positive nodes. Final histologic grading was a pT4aN1 adenosquamous carcinoma (Figure 4). Based on the College of American Pathology system, the Tumor Regression Grade (TRG) 

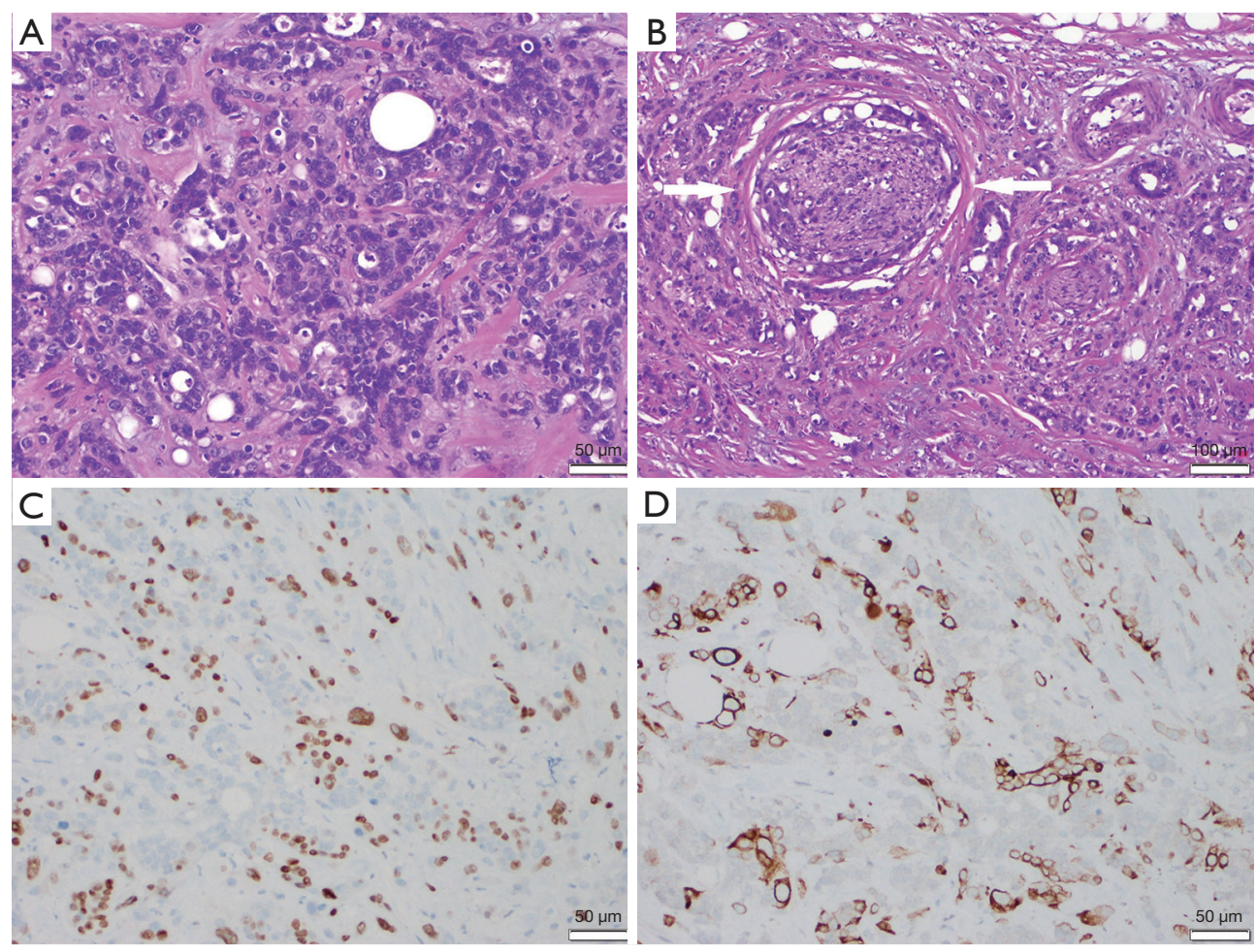

Figure 4 Poorly-differentiated esophageal adenosquamous carcinoma. Tumor composed of solid nests and glandular architecture, involving esophageal muscular wall (A and B, H\&E sections, 20× and 10×, respectively). Note concentric perineural invasion (arrows in B). Immunohistochemical staining showed tumor cells are labeled by squamous markers P40 and CK5/6 (approximately 40\% of cells; C and D, both $20 \times$, respectively). Final pathologic staging pT4aN1.

was designated as a 3 , indicating poor or no response with extensive residual cancer with no evident tumor regression. There was also lymphovascular and perineural invasion.

His post-operative course was predominantly uneventful apart from a mild diastolic dysfunction corrected with diuretics. A routine fluoroscopic assessment confirmed anastomotic integrity and normal emptying of the gastric pull-through. The patient was discharged on post-operative day 7 on a clear liquid diet and a tube feeding regimen. By the 2-week post-operative clinic visit, the patient was tolerating most solid foods and was able to have the jejunostomy removed. To date, he was found to be without residual or recurrent disease by either imaging or serum tumor markers. Given his final stage, future surveillance will consistent of 6-month interval for the initial two years, then annually to follow.

\section{Discussion}

With more active systemic therapies in esophageal cancer
(EC), response assessment has become increasingly important, with changes in metabolic activity correlating with tumor response and prognosis (4). For stage IV patients, recent data supports local therapies in responders, with up to $20 \% 5$-year survival (5). In this case, the patient was deemed to have metabolic evidence of non-regional adenopathy by consensus multidisciplinary opinion since biopsy was not feasible in this location. The reduction in both tumor volume and max SUV on PET/CT serial imaging pre and post chemotherapy indicated that his disease responded significantly, without progression, suggesting he would benefit from local therapy.

The education value of this case lies in the diagnostic pitfall of post-treatment imaging at early post radiation time points. The repeat PET/CT at conclusion of all neoadjuvant therapy was performed 3.5 weeks after the completion of radiotherapy. When the external radiation planning images are registered to the initial and repeat PET/CT exams (Figure 5) it becomes clear that the areas of liver parenchyma receiving the highest dose correlate with 

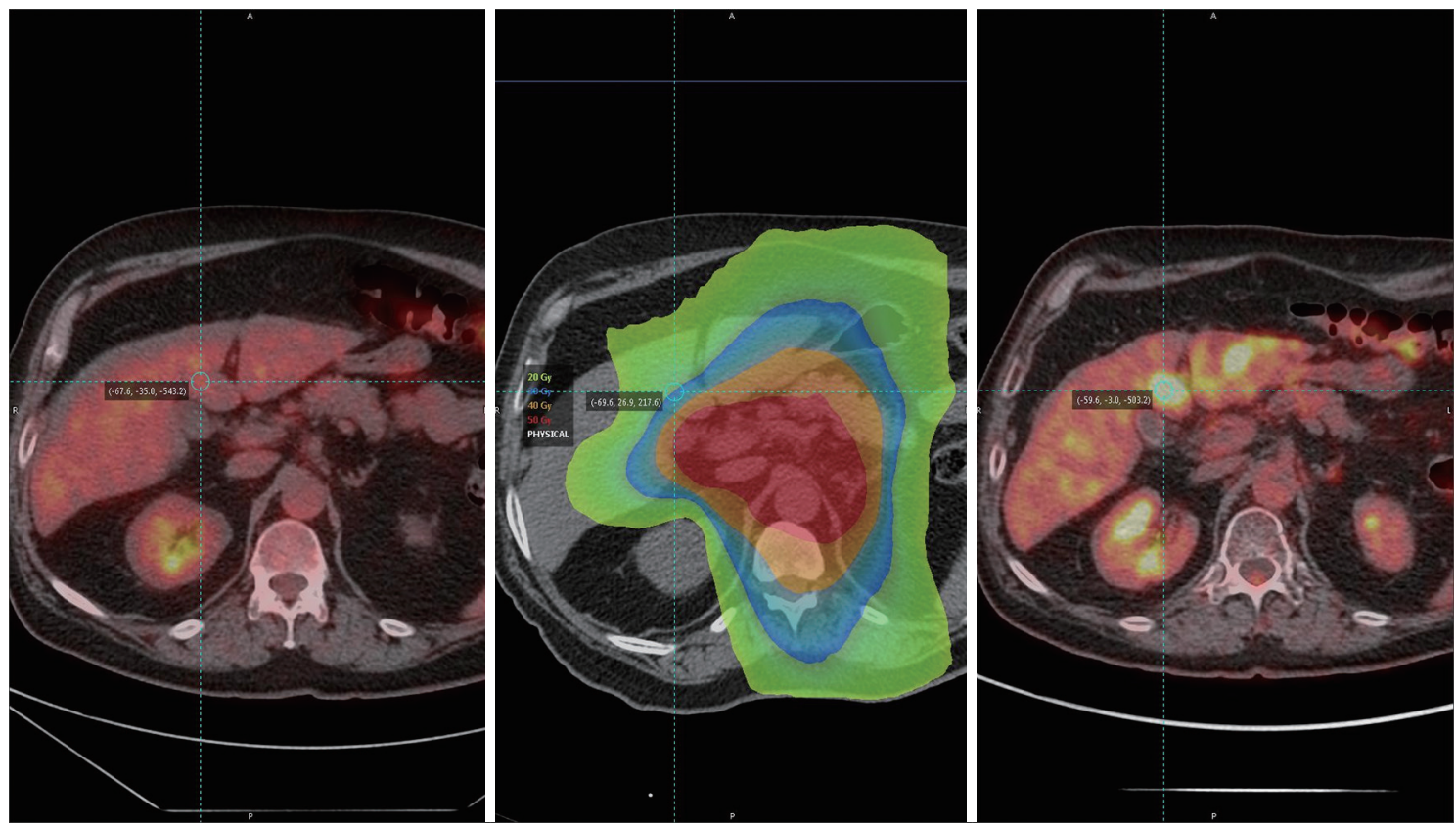

Figure 5 Initial PET (left), XRT planning (middle), repeat PET (right). 30 Gy (blue) and 40 Gy (orange) isodose lines correlate with areas of mass-like hypermetabolism. Largest region was localized for biopsy under ultrasound based on proximity to gallbladder. PET, positron emission tomography; XRT, external beam radiotherapy.

the foci of FDG-uptake.

Imaging PET/CT false positives specific to radiationinduced liver injury in the setting of esophageal carcinoma have been reported (6-8). The causes are multifactorial and include: atypical infections (9), drug-reactions (10), co-existing neoplasms (11), immunotherapy (12), as well as proliferative connective tissue disorders (13). Given the breadth of potential etiologies and the importance of accurate staging, biopsy still holds great clinical value with an acceptable risk-benefit ratio when patient management would change. With an expanding role for consolidation EC radiation in the upper abdomen, knowledge of the potential metabolic benign changes within the imaged radiation field is increasingly important.

\section{Conclusions}

PET/CT remains a valuable tool in both quantifying and following disease during staging, treatment, and surveillance phases of therapy. Clinical context, however, is critical to the evaluation of patients who have recently completed radiation therapy. Improving collaboration between diagnostic radiology and radiation oncology will help prospectively identify such instances by cross-referencing treatment planning data with any areas of unexplained imaging findings leading to improved multidisciplinary coordination of care.

\section{Acknowledgments}

None.

\section{Footnote}

Conflicts of Interest: The authors have no conflicts of interest to declare.

Ethical Statement: The authors are accountable for all aspects of the work in ensuring that questions related to the accuracy or integrity of any part of the work are appropriately investigated and resolved. Informed consent was obtained from the patient for publication of this manuscript and any accompanying images.

\section{References}

1. van Hagen P, Hulshof MC, van Lanshot JJ, et al. Preoperative chemoradiotherapy for esophageal or 
junctional cancer. N Engl J Med 2012;366;2074-84.

2. Lordick F, Ott K, Krause BJ, et al. PET to assess early metabolic response and to guide treatment of adenocarcinoma of the oesophagogastric junction: the MUNICON phase II trial. Lancet Oncol 2007;8;797-805.

3. Venkat PS, Shridnar R, Naghavi AO, et al. Dose escalated neoadjuvant chemoradiotherapy with dose-painting intensity-modulated radiation therapy and improved pathologic complete response in locally advanced esophageal cancer. Dis Esophagus 2017;30;1-9.

4. zum Büschenfelde CM, Herrmann K, Schuster T, et al. (18)F-FDG PET-guided salvage neoadjuvant radiochemotherapy of adenocarcinoma of the esophagogastric junction: the MUNICON II trial. J Nucl Med 2011;52;1189-96.

5. Mizrak Kaya D, Wang X, Harada K, et al. 101 Long-Term Survivors Who Had Metastatic Gastroesophageal Cancer and Received Local Consolidative Therapy. Oncology 2017;93;243-8.

6. DeLappe EM, Truong MT, Bruzzi JF, et al. Hepatic radiation injury mimicking a metastasis on positronemission tomography/computed tomography in a patient with esophageal carcinoma. J Thorac Oncol 2009;4;1442-4.

7. Paudel N, Kunz PL, Poultsides GA, et al. False positive $18 \mathrm{~F}$-fluorodeoxyglucose positron emission tomography/

Cite this article as: Parsee AA, McDonald JA, Jiang K, Latifi K, Mehta R, Frakes JM, Pimiento JM, Hoffe SE. Radiation-induced hepatitis masquerading as metastatic disease: the importance of correlating diagnostic imaging with treatment planning. J Gastrointest Oncol 2020;11(1):133-138. doi: 10.21037/jgo.2019.09.09 computed tomography liver lesion mimicking metastasis in 2 patients with gastroesophageal cancer. Pract Radiat Oncol 2014;4;368-71.

8. Rabe TM, Yokoo T, Meyer J, et al. Radiation-Induced Liver Injury Mimicking Metastatic Disease in a Patient With Esophageal Cancer: Correlation of Positron Emission Tomography/Computed Tomography With Magnetic Resonance Imaging and Literature Review. J Comput Assist Tomogr 2016;40;560-3.

9. Liu B, Dong L, Wang X, et al. Tuberculosis mimicking metastases by malignancy in FDG PET/CT. QJM 2017;110;173-4.

10. Bhatt G, Bhatt A, Dragun AE, et al. Bisphosphonaterelated osteonecrosis of the jaw mimicking bone metastasis. Case Rep Oncol Med 2014;2014:281812.

11. Zhang P, Meng X, Xia L, et al. Non-small cell lung cancer with concomitant intramuscular myxoma of the right psoas mimicking intramuscular metastasis: A case report and literature review. Oncol Lett 2015;10;3059-63.

12. Kalkanis D, Kalkanis A, Gomaa O, et al. F-18 FDG PET positive hilar and mediastinal lymphadenopathy mimicking metastatic disease in a melanoma patient treated with interferon-alpha-2b. Clin Nucl Med 2011;36;154-5.

13. Viswanath L, Pallade S, Krishnamurthy B, et al. DarierRoussy Sarcoidosis Mimicking Metastatic Breast Cancer. Case Rep Oncol 2009;2;251-4. 